
\title{
25 Research Soure \\ Dezocine inhibits cell proliferation, migration and invasion by targeting CRABP2 in ovarian cancer
}

\section{Chuanfeng Zhang}

Shandong Cancer Hospital and Institute, Shandong First Medical University and Shandong Academy of Medical Sciences

\section{Ruirui Pan}

Shandong Cancer Hospital and Institute, Shandong First Medical University and Shandong Academy of Medical Sciences

\section{Shuangshuang $\mathrm{Ma}$}

Shandong Cancer Hospital and Institute, Shandong First Medical University and Shandong Academy of Medical Sciences

\section{Shoucai Xu}

Shandong Cancer Hospital and Institute, Shandong First University and Shandong Academy of Medical Sciences

\section{Baosheng Wang ( $\nabla$ 494365369@qq.com)}

Shandong Cancer Hospital and Institute, Shandong First Medical University and Shandong Academy of Medical Sciences https://orcid.org/0000-0003-0606-2010

\section{Research}

Keywords: Ovarian cancer, Dezocine, Proliferation, Invasion, Apoptosis, CRABP2

Posted Date: March 4th, 2020

DOI: https://doi.org/10.21203/rs.3.rs-16025/v1

License: (c) (i) This work is licensed under a Creative Commons Attribution 4.0 International License. Read Full License 


\section{Abstract}

Background Previous studies have shown that some anesthesia drugs can inhibit tumor growth and metastasis. As a clinical anesthetic drug, dezocine has been reported to play an important role in immune function. However, the effects of dezocine on ovarian cancer cell growth and metastasis are not fully understood.

Results In this study, we found that dezocine dose-dependently inhibited the viability of ES-2 and SKOV3 cells. Dezocine suppressed the migration and invasion abilities of ovarian cancer cells, and promoted apoptosis. Moreover, the Akt/mTOR signaling pathway was also inhibited by dezocine. Furthermore, mechanism study showed that dezocine could significantly inhibited the expression of CRABP2, and CRABP2 overexpression reversed the inhibitory effects of dezocine on ovarian cancer cell proliferation and migration.

Conclusion In conclusion, dezocine has significant anti-tumor effects on the growth and metastatic potential of ovarian cancer cells, and CRABP2 functions as a downstream effector of dezocine.

\section{Introduction}

Ovarian cancer is the most lethal malignancy in gynecology, with high morbidity and mortality $[1,2]$. According to statistics, 239,000 new cases of ovarian cancer occur each year in the world, causing 152,000 deaths $[3,4]$. Most of the early ovarian cancer without obvious symptoms is a major cause of high mortality of ovarian cancer [5-7]. Despite significant advances in surgery and chemotherapy, the prognosis for patients with ovarian cancer is still unsatisfactory. The five-year survival rate for patients with stage I or II ovarian cancer is $80-95 \%$, compared with less than $30 \%$ for advanced patients [8]. Therefore, it is important to clarify the pathological mechanism of ovarian cancer and develop new treatment methods.

Anesthesia methods and drugs have been shown to affect the immune function of tumor patients, and even certain anesthetics can inhibit tumor growth and metastasis [9-11]. For example, sevoflurane has been revealed to function as an anti-tumor reagent in different types of cancer, including ovarian cancer [12-14]. As a representative opioid-receptor agonist/antagonist, dezocine has been widely used in clinical anesthesia and analgesics for postoperative cancer with minimal side effects [15-17]. Feng $C$ et al. previously reported that dezocine could regulate the secretion of IL-12 and IL-10 in dendritic cells, and enhance the activity of T cells during the maturation of dendritic cells, affecting immune function [18]. Wang $\mathrm{F}$ et al. found that dezocine reduces the inhibitory effect of NK cells and CD4 ${ }^{+}$activity and the activity of $\mathrm{CD} 8^{+}$cells in breast cancer patients undergoing radical mastectomy, which is beneficial to the recovery of immune function [19]. These studies suggest that dezocine plays an important role in immune function. Tumor development is usually accompanied by immunodeficiency. However, whether dezocine has a direct effect on tumor cell growth and metastasis has poorly studied. 
Therefore, for the first time, we examined the effect of dezocine on the proliferation, migration and apoptosis of ovarian cancer cells. Our data revealed an anti-tumor activity of dezocine in ovarian cancer, and identified that cellular retinoic acid binding protein 2 (CRABP2) was a downstream effector of dezocine.

\section{Material And Methods}

Cell culture and treatment

Human ovarian cancer cell lines ES-2 and SKOV3 obtained from Cell Bank of Chinese Academy of Sciences (Shanghai, China). Cells were cultured in DMEM medium (Gibco, USA) supplemented with 10\% FBS. Cells were treated with dezocine, and DMSO was used as negative control (NC). The cDNA sequence od CRABP2 was cloned into pcDNA3.1 vector, and the blank pcDNA3.1 was used as control. The plasmids were transfected into ovarian cancer cells by Lipofectamine 2000 (Invitrogen, USA).

Dose-dependent assay

Cells were treated with different concentrations of dezocine $(0,2.5,5,10,20,30,40,50,60,80,120$, and $200 \mu \mathrm{g} / \mathrm{ml}$ ) in a $96-w e l l$ plate at $37^{\circ} \mathrm{C}$ for $24 \mathrm{~h}$, respectively. Then, Cell Counting Kit-8 (CCK-8) reagent (Beijing Solarbio Science \& Technology, Beijing, China) was added in each well and incubated for 90 min. The absorbance at $450 \mathrm{~nm}$ was recorded with a Bio-Rad microplate reader (Bio-Rad, USA).

Cell proliferation assay

Cell proliferation was measured using CCK8 assay. Ovarian cancer cells were exposed to dezocine $\mathrm{n}$ a 96well plate at $37^{\circ} \mathrm{C}$ for $0,24,48$, and $72 \mathrm{~h}$, respectively. The absorbance at $450 \mathrm{~nm}$ was recorded according to the above steps.

Colony formation assay

Following treatment with dezocine for $24 \mathrm{~h}$, cells were seeded into $35 \mathrm{~mm}$-plates with $5 \times 10^{2}$ cells/well and cultured with DMEM at $37^{\circ} \mathrm{C}$ for $1-2$ week. Colonies were fixed with $4 \%$ paraformaldehyde for 30 min and stained with $0.1 \%$ crystal violet for $30 \mathrm{~min}$. The number of colonies was counted under an optical microscope.

Wound-healing assay

When the cells $\left(5 \times 10^{5}\right)$ reached a confluency of $90 \%$ in 6 -well plates, they were scraped by a sterile pipette tip, and were treated with dezocine for $0,24 \mathrm{~h}$. Photographs were taken at the same wound location using an optical microscope (OLYMPUS, Japan), and the width of the wound was measured using ImageJ software (NIH, Bethesda, USA).

Transwell migration and invasion assay 
Cell invasion was analyzed by Transwell chambers $(8.0 \mu \mathrm{m}$; Millipore, MA, USA). After $24 \mathrm{~h}$ of treatment with dezocine or DMSO, cells $\left(1 \times 10^{5}\right)$ were inoculated into the upper chamber precoated with Matrigel (BD Bioscience, CA, USA), and the lower chamber was filled with $700 \mu$ l of medium containing $20 \%$ FBS. After incubation for $24 \mathrm{~h}$, cells in the upper chamber were removed, and then the invaded cells were fixed with $4 \%$ paraformaldehyde and stained with $5 \%$ crystal violet solution for $20 \mathrm{~min}$. For cell migration assays, the Matrigel precoating was not performed. The migrated and invaded cells were photographed under a light microscope (100 × magnification; Nikon, Japan).

Flow cytometry

Apoptosis was detected using the Annexin V-FITC kit (BioVision, USA) according to the manufacturer's instructions. The cells treated with dezocine or DMSO for $24 \mathrm{~h}$ were collected, and incubated in serumfree medium for another $24 \mathrm{~h}$. Thereafter, cells were incubated with Annexin V-FITC and PI for $30 \mathrm{~min}$ at room temperature in the dark. Finally, the percentage of apoptotic cells was measured using a BD FACSCalibur (Beckman Coulter, CA, USA).

Western blotting analysis

RIPA Lysis Buffer (CWBIO, Beijing, China) was used to extract proteins in cells treated with dezocine for $48 \mathrm{~h}$. Following the concentration was determined by BCA kit (CWBIO), the protein samples $(20 \mu \mathrm{g})$ were separated by $10 \%$ SDS-PAGE and transferred onto PVDF members (Millipore, Billerica, MA, USA). After blocked with $5 \%$ dried skimmed milk for $1 \mathrm{~h}$, the members were incubated with primary antibodies at $4{ }^{\circ} \mathrm{C}$ overnight, and then incubated with HRP-conjugated secondary antibodies at room temperature. An enhanced chemiluminescence reagents (CWBIO) was performed to visualize the blot bands. The antibodies, including anti-Bcl-2 (Cat no. 12789-1-AP), anti-Bax (Cat no. 50599-2-lg), anti-p-Akt (Cat no. 66444-1- Ig), anti-mTOR (Cat no. 20657-1-AP), anti-GAPDH (Cat no. 10494-1-AP), and anti-CRABP2 (Cat no. 10225-1-AP) were obtained from Proteintech Group (IL, USA); anti-cleaved Caspase 3 (Cat no. 9661), anti-Akt (Cat no. 9272), anti-p-mTOR (Cat no. 5536), anti-p70s6k (Cat no. 9204), and secondary antibodies were obtained from Cell Signaling Technology (Danvers, USA).

Statistical analysis

The values were presented as Mean \pm SD and statistical analyzed using GraphPad Prism software. Differences between groups was assessed using Student's t-test or one-way ANOVA followed by Dunnett's test. P-values less than 0.05 indicate a significant difference.

\section{Results}

Dezocine inhibits ovarian cancer cell proliferation and colony formation

To determine the cytotoxicity of dezocine to ovarian cancer, ES-2 and SKOV3 cells were treated different concentrations of dezocine $(0,2.5,5,10,20,30,40,50,60,80,120$, and $200 \mu \mathrm{g} / \mathrm{ml})$. As shown in Fig. 1A 
and $B$, the viability was significantly reduced in a dose-dependent manner after treated with $10 \mu \mathrm{g} / \mathrm{ml}$ or higher concentrations of dezocine for $24 \mathrm{~h}$ in both ES-2 and SKOV3 cells. The IC ${ }_{50}$ of dezocine for ES- 2 cells was $26.42 \mu \mathrm{g} / \mathrm{ml}$, and it was for SKOV3 cells was $29.32 \mu \mathrm{g} / \mathrm{ml}$. Therefore, ES-2 cells were treated with $15.85 \mu \mathrm{g} / \mathrm{ml}$ of dezocine was used for appropriate suppression effect in subsequent experiments, and SKOV3 cells were treated with dezocine at $17.59 \mu \mathrm{g} / \mathrm{ml}$. Moreover, CCK8 assay showed that ES-2 and SKOV3 cell viability was dramatically reduced by dezocine in a time-dependent manner (Fig. 1C and D). As evident from colony formation assay, compared with the NC group, the colony-forming abilities of ES2 and SKOV3 cells were greatly inhibited after exposure to dezocine (Fig. 1E-G). These results indicate the growth-inhibitory effect of dezocine on ovarian cancer.

Dezocine suppresses the migration and invasion of ovarian cancer cells

To determine the effect of dezocine on the mobility of ovarian cancer cells, the migration and invasion abilities of ES-2 and SKOV3 cells after exposure to dezocine were evaluated using wound-healing and transwell assays. As obvious from the wound-healing assay, compared with corresponding control group, the migration ability of ES-2 and SKOV3 cells exposed to dezocine was significantly inhibited (Fig. 2A). The migration-inhibitory effect of dezocine were further examined by transwell assay. As shown in Fig. 2B, the number of migrated cells in dezocine-treated group was significantly lower than that in control group. Further, transwell invasion assay indicated that the invasion abilities of ES-2 and SKOV3 cells were significantly repressed by dezocine (Fig. 2C). Our findings reveal that dezocine possesses the activity to inhibit the metastatic potential of ovarian cancer cells.

Dezocine promotes apoptosis of ovarian cancer cells and inhibits the Akt/mTOR signaling pathway

Flow cytometry assay was used to measure the effect of dezocine on apoptosis of ovarian cancer cells. As shown in Fig. 3A, dezocine significantly increased the proportion of apoptosis in ES-2 and SKOV3 cells compared with the control group. Moreover, the expression of apoptosis-related proteins was detected to investigate the molecular mechanism underlying the induced apoptosis by dezocine. As displayed in Fig. 3B, the expression of anti-apoptotic protein Bcl-2 was prominently down-regulated by dezocine in both ES-2 and SKOV3 cells, while the expression of pro-apoptotic proteins Bax and cleaved Caspase 3 was prominently enhanced in dezocine-treated cells. Collectively, dezocine may induce apoptosis of ovarian cancer cells by regulating the Bcl-2/Bax axis and Caspase 3 activity.

Considering the key role of Akt/mTOR signaling pathway in regulating cell proliferation, migration and apoptosis, we examined the effect of dezocine on the key components of the signaling pathway. As indicated by western blot analysis, we found that the total expression of Akt and mTOR was not affected by dezocine, whereas the expression of $\mathrm{p}$-Akt and p-mTOR was markedly reduced in dezocine-treated group (Fig. 3C). Consistently, the expression of p70S6K, an important downstream of p-mTOR, was downregulated by dezocine in ovarian cancer cells. Therefore, dezocine may exert anti-cancer activity by inhibiting activation of the Akt/mTOR signaling pathway in ovarian cancer.

Dezocine inhibits the proliferation and migration of ovarian cancer by down-regulating CRABP2 
To further investigate the molecular mechanism underlying the anti-cancer activity of dezocine in ovarian cancer, RNA-Seq was performed. As shown in Fig. 4A, dezocine treatment resulted in multiple differential expressed genes (DEGs; $\mid \log _{2}$ fold changel>1 \& Q value < 0.001) in ES-2 cells, 259 genes were significantly up-regulated and 115 genes were down-regulated. Hierarchical clustering analysis of DEGs was shown in Supplementary Fig. 1A, dezocine led to different gene expression patterns in ES-2 cells compared with NC group. The classification and statistics of KEGG biological pathways of DEGs were shown in Supplementary Fig. 1B, dezocine affected multiple cellular functional pathways.

In order to further search for the downstream effectors of dezocine, 6 genes (GAGE2E, CDRT4, IGFBP5, CRABP2, FLT1, and KIF20A) down-regulated by dezocine were selected as candidates. RT-PCR analysis further demonstrated that CRABP2 expression was most significantly down-regulated by dezocine in ES-2 cells (Supplementary Fig. 1C). Similarly, there was a significant decrease in CRABP2 expression in dezocine-treated SKOV3 cells (Fig. 4B). Accordingly, the expression of CRABP2 protein was significantly inhibited by dezocine in both ES-2 and SKOV3 cells, while CRABP2 overexpression could partially recovered its expression (Fig. 4C). Therefore, CRABP2 was a downstream effector of dezocine in ovarian cancer. Further study showed that CRABP2 overexpression reversed the inhibitory effect of dezocine on the proliferation of both ES-2 and SKOV3 cells (Fig. 4D, E). Transwell assay showed that the depression in cell migration induced by dezocine was significantly rescued by CRABP2 overexpression in ovarian cancer cells (Fig. 4F, G). Collectively, these results suggest that dezocine might inhibit the proliferation and migration of ovarian cancer cells by targeting CRABP2.

\section{Discussion}

Emerging evidence has reported that dezocine could regulate immune function of cancer patients after surgery [19]. In this study, we investigated the anti-tumor activity of dezocine on ovarian cancer cells for the first time and demonstrated that dezocine could inhibited the viability of ES-2 and SKOV3 cells in a dose-independent manner. Moreover, treatment with dezocine suppressed the migration and invasion abilities of ovarian cancer cells. Additionally, dezocine was been shown to induce apoptosis in ovarian cancer cells and regulate the expression of Bcl-2/Bax and cleaved Caspase 3. Taken together, our data indicate that dezocine functions as a potential anti-tumor reagent in the therapy of ovarian cancer.

It is generally known that the Akt/mTOR signaling pathway, as a classic signaling pathway, is frequently activated in tumors and plays a key role in the malignant transformation of tumors, including ovarian cancer $[20,21]$. Overactivation of the Akt/mTOR pathway is closely associated with tumorigenesis, tumor progression and drug resistance $[22,23]$. Therefore, inhibiting activation of this signaling pathway is regarded as a promising method to control the growth and metastatic ability of tumors $[24,25]$. Many antitumor drugs could inhibit the activation of this signaling pathway [26, 27]. In the present study, our data demonstrated that the expression of p-Akt and p-mTOR was markedly reduced with dezocine treatment, suggesting that dezocine could suppress activation of the Akt/mTOR signaling pathway in ovarian cancer cells. Taken together, these data indicate that the anti-tumor activity of dezocine may be associated with the inhibition of the Akt/mTOR signaling pathway. 
CRABP2, a member of the intracellular lipid-binding proteins family, has been showed to transport RA to the retinoic acid receptor (RAR) in the nucleus $[28,29]$. Increasing study report that dysregulated CRABP2 is associated with the progression of tumors [30]. CRABP2 is highly expressed in pancreatic ductal adenocarcinoma [30], breast cancer [31] and non-small cell lung cancer (NSCLC) [32], its high expression is associated with poor prognosis of patients with NSCLC or ER-negative breast cancer [32, 33]. Yu S et al. report that CRABP2 promotes the invasion of pancreatic cancer cells through stabilizing the interleukin 8 expression [34]. Wu $\mathrm{J}$ et al. demonstrate that CRABP2 enhances lung cancer metastasis by HuR and integrin $\beta 1 / F A K / E R K$ signaling [35]. These results highlight the oncogenic role of CRABP2 in the progression of cancers. However, the role of CRABP2 in breast cancer growth and metastasis has been interpreted differently. In ER + breast cancer cells, CRABP2 suppresses tumor metastasis by inhibiting ubiquitination of Lats1; while CRABP2 enhances ubiquitination of Lats1 and promotes tumor metastasis [31]. Toyama A et al. show that CRABP2 is strongly expressed in serous carcinoma, which id identified as a candidate subtype-specific biomarker for ovarian cancers [36]. In this study, we found that CRABP2 was markedly inhibited by dezocine in ovarian cancer cells at both mRNA and protein levels. Moreover, CRABP2 overexpression could partially reverse the inhibitory effect of dezocine on cell proliferation and migration. Taken together, CRABP2 might exert an oncogenic role in ovarian cancer and function as a downstream effector of dezocine.

In the present study, our findings identified the anti-cancer activity of dezocine in ovarian cancer, dezocine could inhibit cell proliferation and invasion, and promote apoptosis. Moreover, mechanism study suggested that CRABP2 was a downstream effector of dezocine, which is involved in the anti-cancer activity of dezocine. Dezocine may represent a promising novel targeted agent in ovarian cancer.

\section{Declarations}

\section{Acknowledgements}

Not applicable.

\section{Authors' contributions}

Conception and design, acquisition of data, or analysis and interpretation of data: all authors. Drafting the article or revising it critically for important intellectual content: CZ, RP and BW. Final approval of the version to be published: all authors. All authors read and approved the final manuscript.

\section{Funding}

No.

\section{Availability of data and materials}

The data supporting the conclusions of this paper are included within the manuscript. 
Ethics approval and consent to participate

Not applicable.

Consent for publication

All the authors agree to the publication clause.

Competing interests

The authors declare that they have no competing interests.

Author details

Shandong Cancer Hospital and Institute, Shandong First Medical University and Shandong Academy of Medical Sciences, Jinan 250117, China.

\section{References}

1. Smolle E, Taucher V, Petru E, Haybaeck J: Targeted treatment of ovarian cancer-the multiple - kinase - inhibitor sorafenib as a potential option. Anticancer Res 2014, 34(4):1519-1530.

2. Harter P, Gershenson D, Lhomme C, Lecuru F, Ledermann J, Provencher DM, Mezzanzanica D, Quinn M, Maenpaa J, Kim J-W et al: Gynecologic Cancer InterGroup (GCIG) consensus review for ovarian tumors of low malignant potential (borderline ovarian tumors). Int J Gynecol Cancer 2014, 24(9 Suppl 3):S5-8.

3. Reid BM PJ, Sellers TA: Epidemiology of ovarian cancer: a review. Cancer Biol Med 2017, 14:9-32.

4. Wang V, Cheng L, Ming L, William W, Deborah B, Yuk-Fu W, Ross B, Samuel CM, Christina AB: Ovarian cancer is a heterogeneous disease. Cancer Genet Cytogenet 2005, 161(2):170-173.

5. Fishman DA, Cohen L, Blank SV, Shulman L, Singh D, Bozorgi K, Tamura R, Timor-Tritsch I, Schwartz PE: The role of ultrasound evaluation in the detection of early-stage epithelial ovarian cancer. Am J Obstet Gynecol 2005, 192(4):1214-1221; discussion 1221-1212.

6. Goonewardene TI, Hall MR, Rustin GJS: Management of asymptomatic patients on follow-up for ovarian cancer with rising CA-125 concentrations. Lancet Oncol 2007, 8(9):813-821.

7. Reade CJ RJ, Busse JW: Risks and benefits of screening asymp-tomatic women for ovarian cancer: a system-atic review and meta-analysis. Gynecol Oncol 2013, 130:674-681.

8. Fowler J, Lucas FAS, Sivakumar S, Deshpande A, Scheet PA: Abstract 2594: Optimizing the replication of cancer genomics workflows: case studies. Cancer Research 2017, 77(13 Supplement):2594-2594.

9. Cakmakkaya OS, Kolodzie K, Apfel CC, Pace NL: Anaesthetic techniques for risk of malignant tumour recurrence. Cochrane Database Syst Rev 2014, 11(11):CD 008877. 
10. Chen WK, Ren L, Wei Y, Zhu D-X, Miao C-H, Xu J-M: General anesthesia combined with epidural anesthesia ameliorates the effect of fast-track surgery by mitigating immunosuppression and facilitating intestinal functional recovery in colon cancer patients. International Journal of Colorectal Disease 2015, 30(4):475-481.

11. Liang H, Yang CX, Zhang B, Zhao ZL, Zhong JY, Wen XJ: Sevoflurane attenuates platelets activation of patients undergoing lung cancer surgery and suppresses platelets-induced invasion of lung cancer cells. Journal of Clinical Anesthesia 2016, 35:304-312.

12. Yang Y, Hu R, Yan J, Chen Z, Lu Y, Jiang J, Jiang H: Sevoflurane inhibits the malignant potential of head and neck squamous cell carcinoma via activating the hypoxia-inducible factor-1a signaling pathway in vitro. Int J Mol Med 2018, 41(2):995-1002.

13. Liang H, Gu M, Yang C, Wang H, Wen X, Zhou Q: Sevoflurane inhibits invasion and migration of lung cancer cells by inactivating the p38 MAPK signaling pathway. J Anesth 2012, 26(3):381-392.

14. Zhang CF WB, Wang XQ, Sheng XG, Cui YC: Sevoflurane inhibits the progression of ovarian cancer through down-regulating stanniocalcin 1 (STC1). Cancer Cell Int 2019, 19(339).

15. Wu L, Dong YP, Sun L, Sun L: Low Concentration of Dezocine in Combination With Morphine Enhance the Postoperative Analgesia for Thoracotomy. J Cardiothorac Vasc Anesth 2015, 29(4):950954.

16. Li T-F, Wu H-Y, Wang Y-R, Li X-Y, Wang Y-X: Molecular signaling underlying bulleyaconitine A (BAA)induced microglial expression of prodynorphin. In: Sci Rep. vol. 7; 2017: 45056.

17. Wang L, Liu X, Wang J, Sun Y, Zhang G, Liang L: Comparison of the efficacy and safety between dezocine injection and morphine injection for persistence of pain in Chinese cancer patients: a metaanalysis. Biosci Rep 2017, 37(3).

18. Feng C, Feng M, Jiao R, Liu D, Jin Y, Zhao X, Xiao R: Effect of Dezocine on IL-12 and IL-10 secretion and lymphocyte activation by culturing dendritic cells from human umbilical cord blood. Eur $J$ Pharmacol 2017, 796:110-114.

19. Fei Wang XZ, Huihui Wang, and Yi Liu: Effects of dezocine and sufentanyl for postoperative analgesia on activity of NK, CD4+ and CD8+ cells in patients with breast cancer. Oncol Lett 2019, 17(3):3392-3398.

20. Lee HJ VGSV, Kim SM, Ha SE, Raha S, Lee WS, Kim EH, Lee SJ, Heo JD and Kim GS: Pectolinarigenin inनduced cell cycle arrest, autophagy, and apop-tosis in gastric cancer cell via PI3KJAKT/mTOR signaling pathway. Nutrients 2018, 10.

21. Aziz A, Farid S, Qin K, Wang H, Liu B: Pim kinases and their relevance to the PI3K/AKT/mTOR pathway in the regulation of ovarian cancer. Biomolecules 2018, 8:7.

22. Kato T, Hagiyama M, Takashima Y, Yoneshige A, Ito A: Cell adhesion molecule-1 shedding induces apoptosis of renal epithelial cells and exacerbates human nephropathies. Am J Physiol Renal Physiol 2018, 314(3):F388-F398.

23. Yan X, Shan Z, Yan L, Zhu Q, Liu L, Xu B, Liu S, Jin Z, Gao Y: High expression of Zinc-finger protein Xlinked promotes tumor growth and predicts a poor outcome for stage II/III colorectal cancer patients. 
Oncotarget 2016, 7(15):19680-19692.

24. Bellacosa A, Kumar CC, Di Cristofano A, Testa JR: Activation of AKT kinases in cancer: implications for therapeutic targeting. Adv Cancer Res 2005, 94:29-86.

25. Porta C, Paglino C, Mosca A: Targeting PI3K/Akt/mTOR Signaling in Cancer. In: Front Oncol. vol. 4; 2014: 64.

26. Xueke Liu CX, Amin Li, Yinci Zhang, Xinkuang Liu, Shuping Zhou, Jing Shen, Zhen Huo, Weiya Cao, Yongfang Ma, Ruyue Xu, Yingru Xing, Yinghai Xie, Shuyu Cai, and Xiaolong Tang: BEZ235 enhances chemosensitivity of paclitaxel in hepatocellular carcinoma through inhibiting the PI3K/Akt/mTOR pathway. Am J Transl Res 2019, 11(12):7255-7271.

27. Ni T, Wang H, Li D, Tao L, Lv M, Jin F, Wang W, Feng J, Qian Y, Sunagawa M: Huachansu Capsule inhibits the proliferation of human gastric cancer cells via Akt/mTOR pathway. Biomedicine \& pharmacotherapy $=$ Biomedecine \& pharmacotherapie 2019, 118:109241.

28. Noy, Noa: Retinoid-binding proteins: mediators of retinoid action. Biochemical Journal 2000, 348(3):481-495.

29. Noy, Noa: Between Death and Survival: Retinoic Acid in Regulation of Apoptosis. Annual Review of Nutrition 2010, 30(1):201-217.

30. Xiao W, Hong H, Awadallah A, Yu S, Zhou L, Xin W: CRABP-II is a highly sensitive and specific diagnostic molecular marker for pancreatic ductal adenocarcinoma in distinguishing from benign pancreatic conditions. Human Pathology 2014, 45(6):1177-1183.

31. Feng X ZM, Wang B, Zhou C, Mu Y, Li J, Liu X, Wang Y, Song Z, Liu P: CRABP2 regulates invasion and metastasis of breast cancer through hippo pathway dependent on ER status. J Exp Clin Cancer Res 2019, 38(1):361.

32. Favorskaya KY, Chemeris G, Komelkov A, Zborovskaya, Tchevkina E: Expression and clinical significance of CRABP1 and CRABP2 in non-small cell lung cancer. Tumour Biol 2014, 35(10):1029510300.

33. Geiger T, Madden SF, Gallagher WM, Cox J, Mann M: Proteomic Portrait of Human Breast Cancer Progression Identifies Novel Prognostic Markers. Cancer Research 2012, 72(9):2428-2439.

34. Yu S, Parameswaran N, Li M, Wang Y, Jackson MW, Liu H, Xin W, Zhou L: CRABP-Il enhances pancreatic cancer cell migration and invasion by stabilizing interleukin 8 expression. In: Oncotarget. vol. 8; 2017: 52432-52444.

35. Wu JI LY, Tseng CW, Chen HJ, Wang LH: Crabp2 Promotes Metastasis of Lung Cancer Cells via HuR

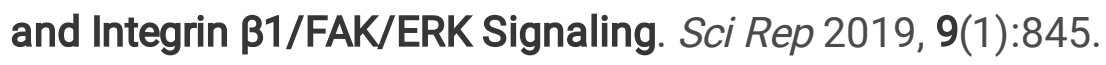

36. Toyama A, Suzuki A, Shimada T, Aoki C, Aoki Y, Umino Y, Nakamura Y, Aoki D, Sato T-A: Proteomic characterization of ovarian cancers identifying annexin-A4, phosphoserine aminotransferase, cellular retinoic acid-binding protein 2, and serpin B5 as histology-specific biomarkers. Cancer Sci 2012, 103(4):747-755.

\section{Supplementary Figure}


(A) Hierarchical clustering analysis of differentially expressed genes in dezocine-treated ES-2 cells. (B) Classification and statistics of KEGG biological pathways of the differentially expressed genes in dezocine-treated cells. (C) Expression of possible downstream effector genes (GAGE2E, CDRT4, IGFBP5, CRABP2, FLT1, and KIF20A) for dezocine in ES-2 cells. ${ }^{*} \mathrm{P}<0.05,{ }^{* *} \mathrm{P}<0.01$.

\section{Figures}

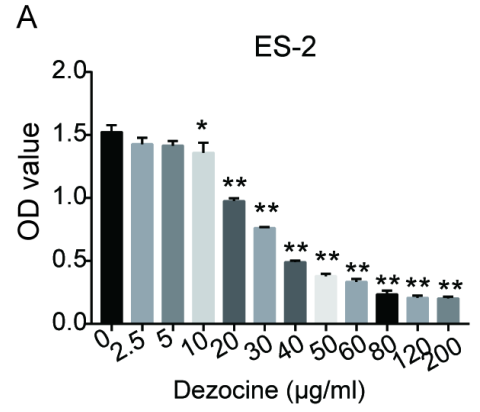

$\mathrm{E}$

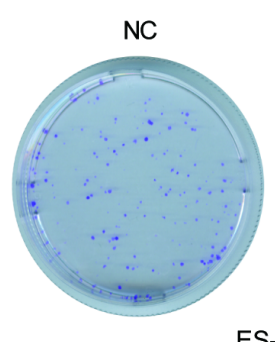

B

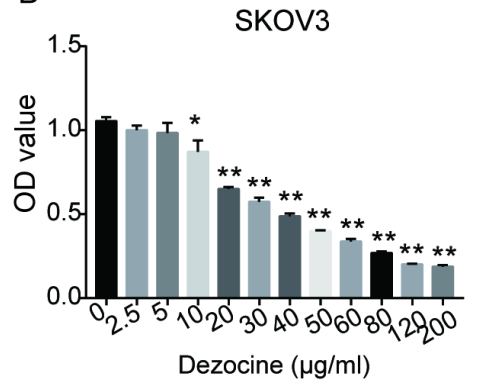

$\mathrm{C}$

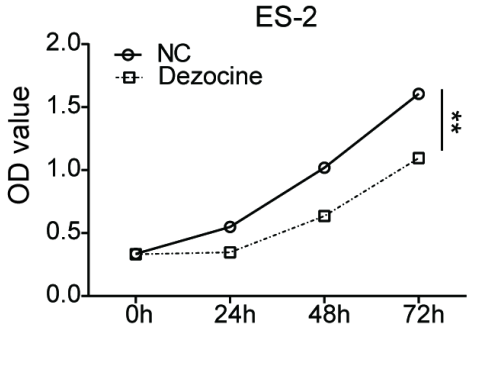

$\mathrm{D}$

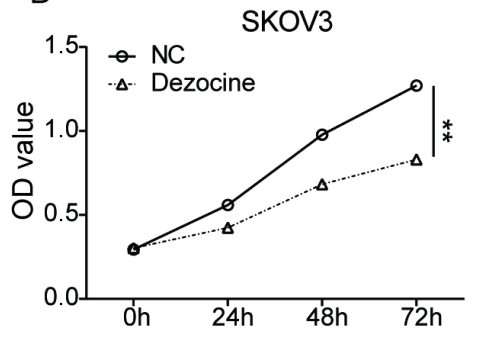

Dezocine

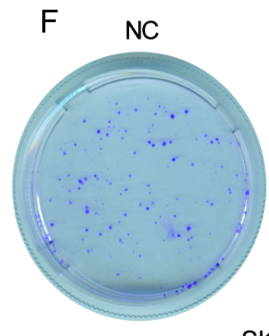

SKOV3

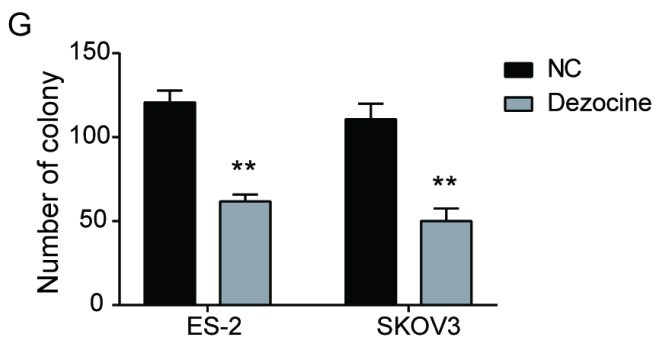

Figure 1

Dezocine inhibits ovarian cancer cell proliferation and colony formation (A and B) ES-2 (A) and SKOV3 (B) cells were treated with various concentration levels of dezocine $(0,2.5,5,10,20,30,40,50,60,80$, 120 , and $200 \mu \mathrm{g} / \mathrm{ml}$ ) for $24 \mathrm{~h}$, and cell viability was measured by CCK8 assay. (C and D) Cell viability was measured by CCK8 assay in ES-2 (C) and SKOV3 (D) cells treated with dezocine for $0,24,48$, and $72 \mathrm{~h}$, respectively. ( $E$ and $F$ ) Colony formation assay showing inhibitory effects of dezocine on ES-2 (E) and SKOV3 (F) cells. (G) Quantitative analysis of colony formation results. ${ }^{*} P<0.05,{ }^{\star *} \mathrm{P}<0.01$. 


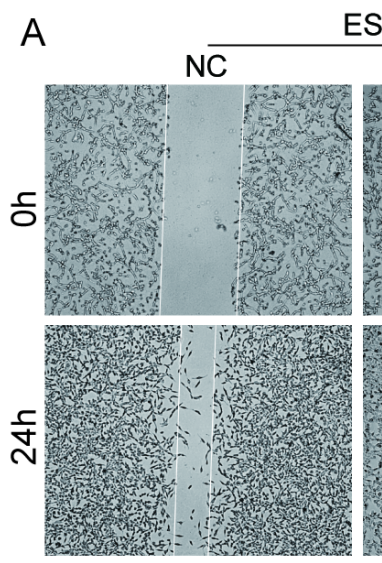

ES-2
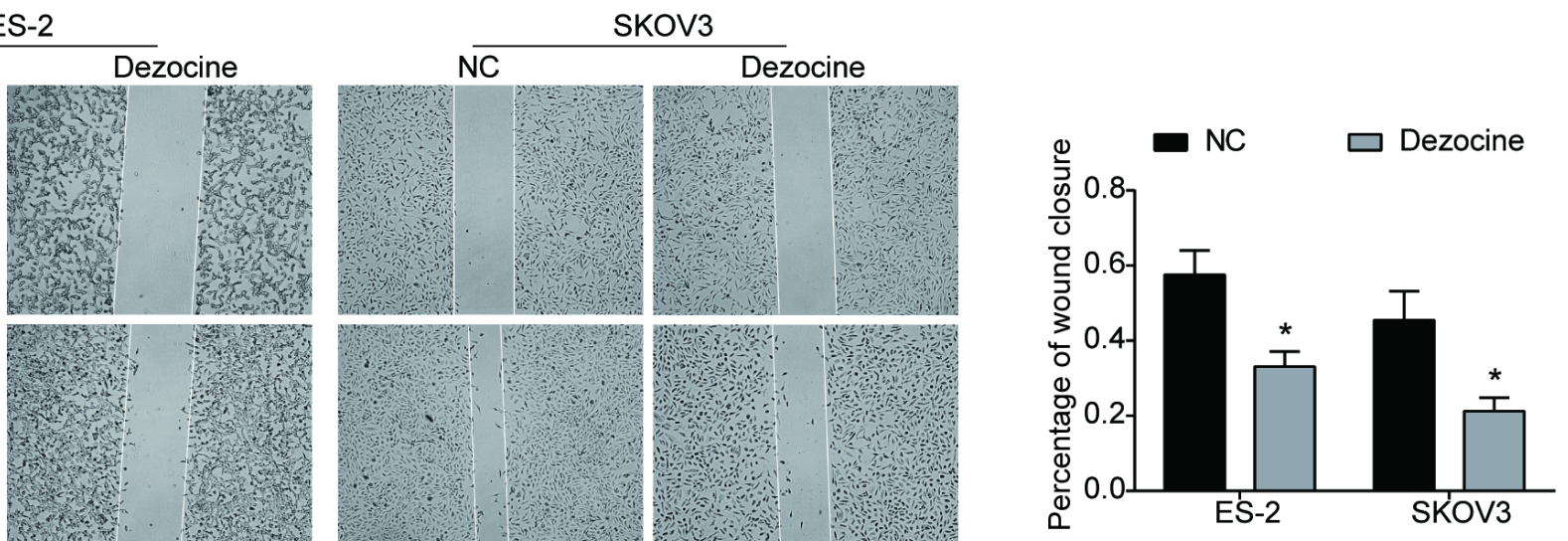

B

ES-2
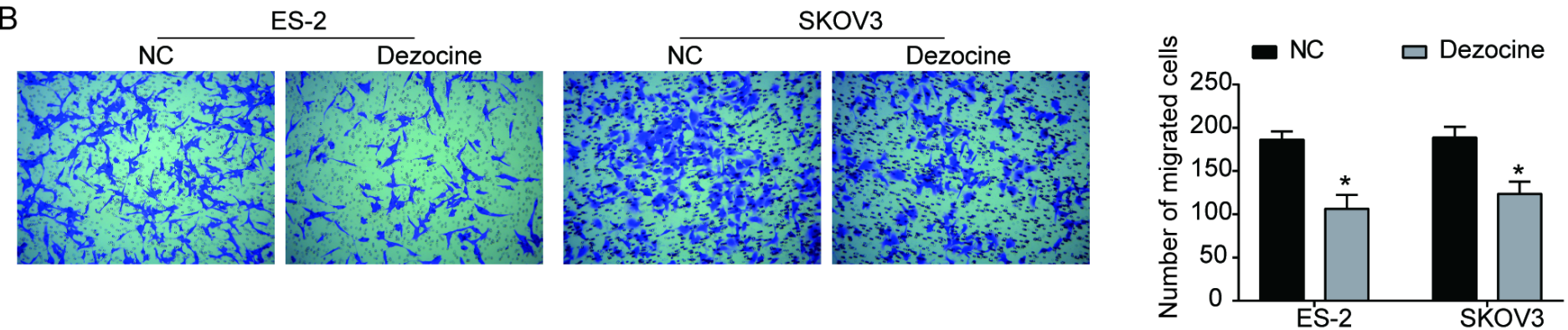

C

ES-2
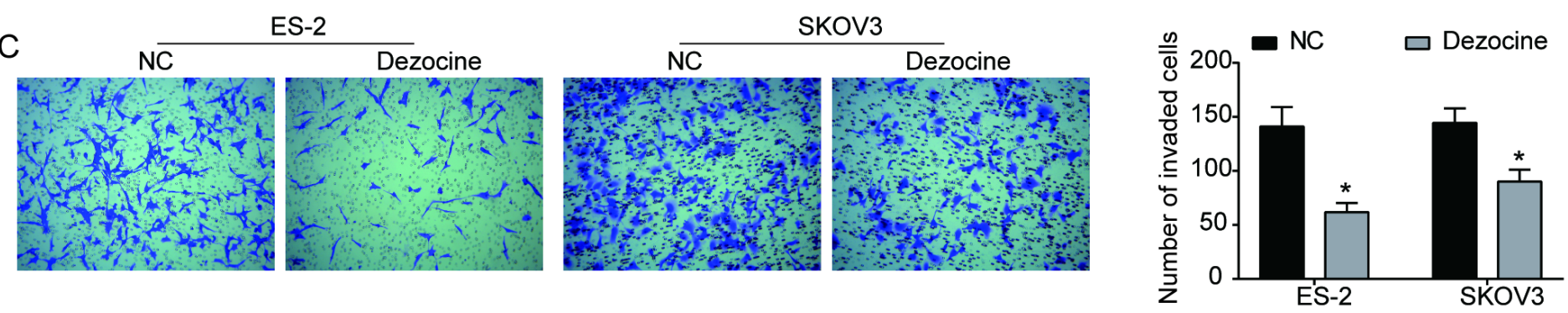

Figure 2

Dezocine suppresses the migration and invasion of ovarian cancer cells (A) After cells treated with dezocine for 0 and $24 \mathrm{~h}$, wound-healing assay was performed to measure the wound closure. (B and $C$ ) Cell migration (B) and invasion (C) were measured using Transwell assay in ES-2 and SKOV3 cells after treated with dezocine for $24 \mathrm{~h}$. ${ }^{*} \mathrm{P}<0.05$. 
A

ES-2

SKOV3
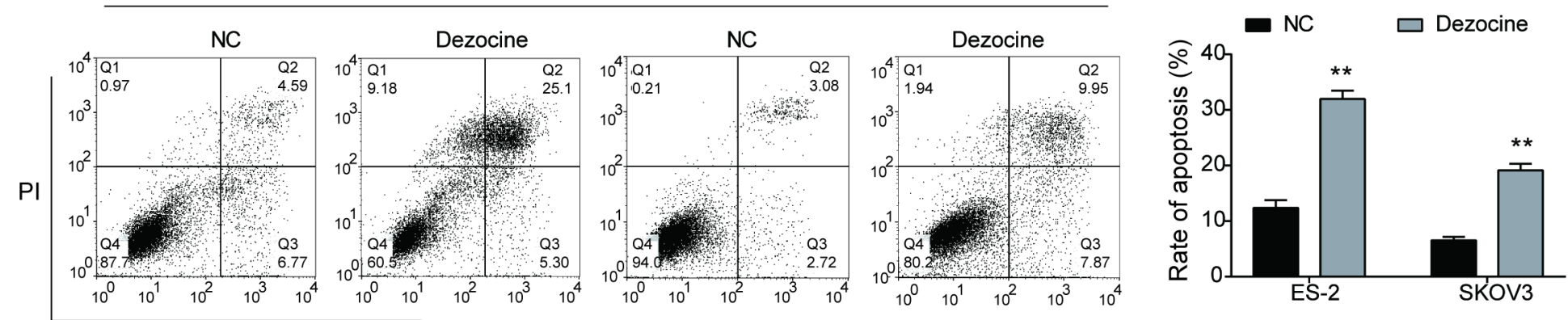

Annexin V/FITC

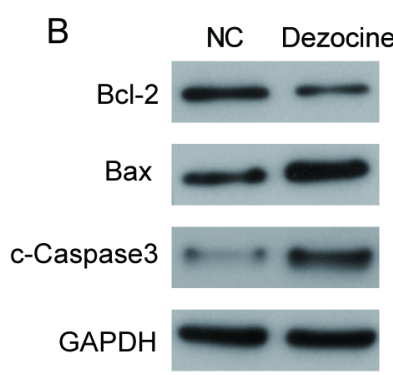

ES-2

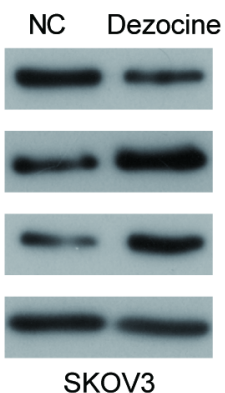

C

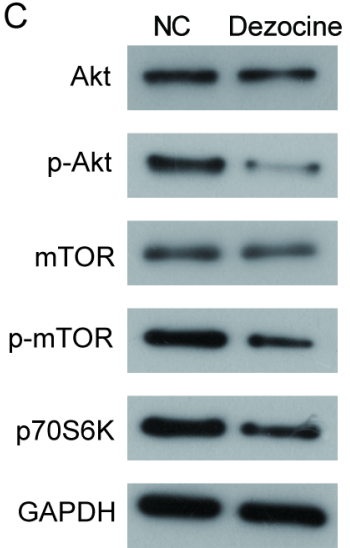

ES-2

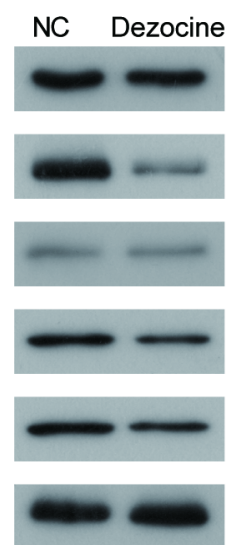

SKOV3
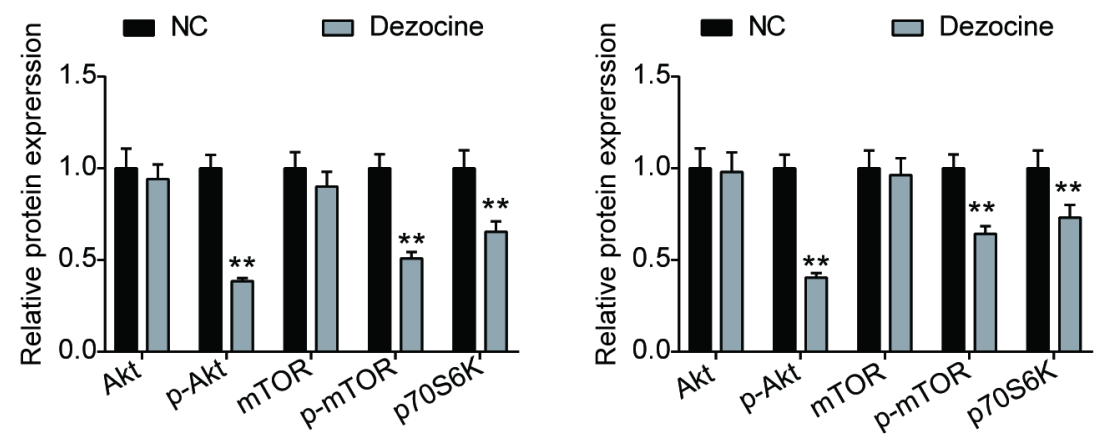

Figure 3

Dezocine promotes apoptosis of ovarian cancer cells and inhibits the Akt/mTOR signaling pathway (A) Cells were treated with dezocine for $24 \mathrm{~h}$, the percentage of apoptotic cells was analyzed by flow cytometry. (B) Western blot analysis of the expression of apoptosis-related proteins, Bcl-2, Bax and cleaved Caspase 3 in ES-2 and SKOV3 cells treated with dezocine. (C) Expression of important components involved in the Akt/mTOR signaling pathway in ES-2 and SKOV3 cells, including Akt, p-Akt, mTOR, p-mTOR, and p70s6k. **P<0.01. 

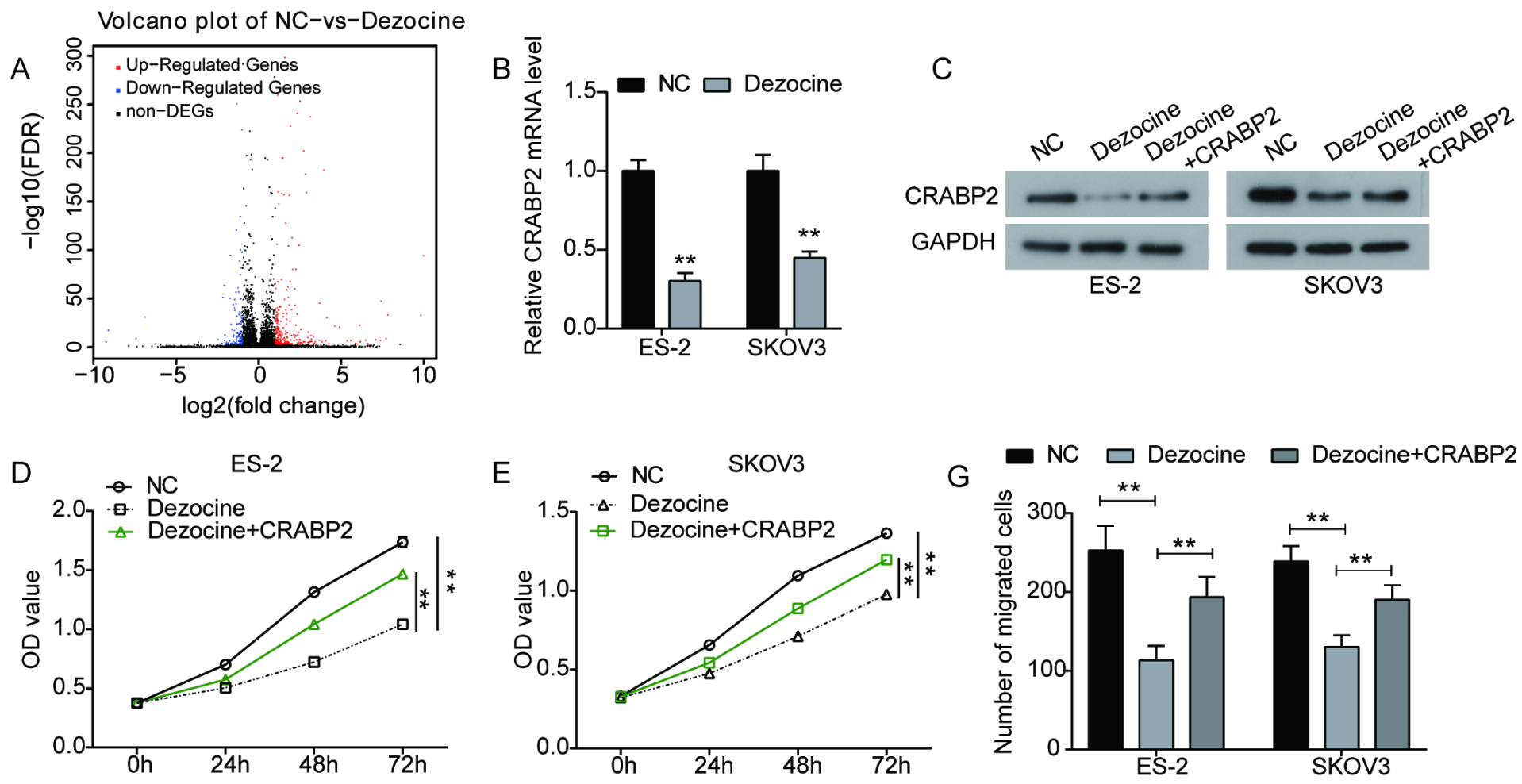

$\mathrm{F}$
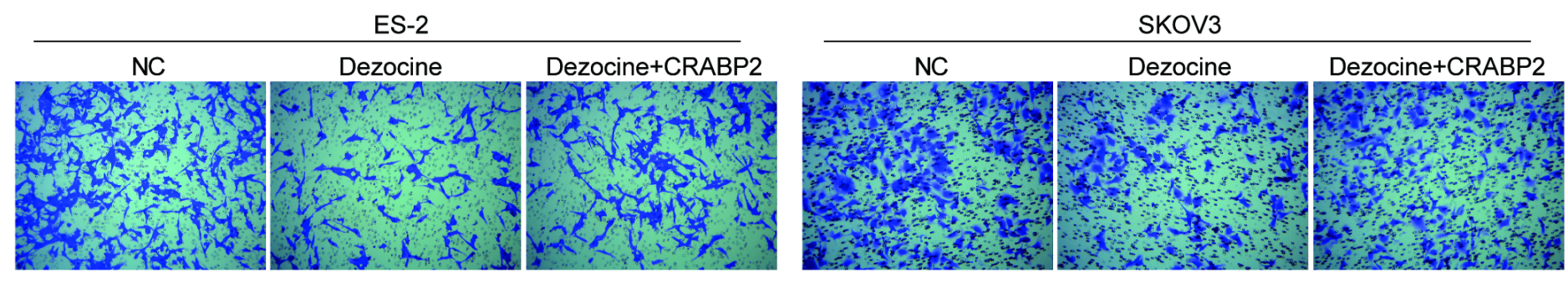

\section{Figure 4}

Dezocine inhibits the proliferation and migration of ovarian cancer by down-regulating CRABP2 (A) By RNA-Seq analysis, the differential expressed genes (DEGs; |log2 fold changel>1 \& Q value $<0.001$ ) in ES-2 cells treated with dezocine. (B) Expression of CRABP2 mRNA was detected by RT-PCR analysis in ES-2 and SKOV3 cells treated with dezocine. (C) Expression of CRABP2 protein was detected by Western blot analysis in ES-2 and SKOV3 cells treated with dezocine or dezocine+pcDNA3.1-CRABP2. (D and E) ES-2 (D) and SKOV3 (E) cells were treated with dezocine or dezocine+pcDNA3.1-CRABP2, and cell viability was measured by CCK8 assay. (F) After indicated treatment, cell migration was measured by Transwell assay. $\star * P<0.01$.

\section{Supplementary Files}

This is a list of supplementary files associated with this preprint. Click to download.

- Supplementaryfigure1.tif 